\title{
Performance of Uplink Random-Access and Downlink TDMA Techniques for Packet Satellite Networks
}

\author{
GIULIANO BENELLI, ENRICO DEL RE, SENIOR MEMBER, IEEE, ROMANO FANTACCI, \\ STUDENT MEMBER, IEEE, AND FRANCESCO MANDELLI
}

In this paper the integration of ALOHA random-access schemes and TDMA techniques in a multibeam packet satellite network are analyzed. The uplink channels operate according to a slottedALOHA protocol, while the downlink channels use a TDMA strategy. Two different models are described. In the first model, each transponder on-board the satellite is permanently connected to a preassigned spot beam. The second model uses a number of transponders lower than the number of spot beams. Some different strategies of transmission are proposed in the latter model, able to provide full interconnection among earth stations by channel-hopping capability of the transponders. The performance of the models is evaluated through queueing theory analysis and extensive computer simulations. The obtained results suggest the second model as an efficient candidate for the implementation of a multibeam packet satellite network. In that case, the system parameters must be carefully chosen in order to avoid possible instability conditions.

\section{INTRODUCTION}

Satellites using multiple-beam antennas and on-board processing capabilities present many attractive features [1]-[3]. The earth coverage by means of narrow spot beams permits higher energy concentration with respect to a wide-area coverage beam and the reuse of the same frequencies in different beams. At the same time, the on-board processing and switching permit more complex and efficient strategies for the traffic routing. In particular, Satellite-Switched Time-Division Multiple Access (SS/ TDMA) systems have been proposed as very efficient satellite communication techniques [4]-[9]. In an S5/TDMA system, the on-board spot beam antennas cover different and spatially disjoint areas. A switching matrix on-board the satellite provides the necessary connections between the uplink and downlink beams, according to the traffic demands. The switching strategy is known at each time instant to the earth stations, which transmit and receive accordingly. The switching strategy and, therefore, the algorithm which determines the time slot assignments, influence significantly the performance of an SS/TDMA system; many different algorithms have been described in the literature [6]-[10].

The TDMA structure, proposed in these systems, is particularly efficient when the traffic has a continuous flow

\footnotetext{
Manuscript received April 6, 1984; revised June 22, 1984. This work was partly supported by CNR (National Research Council, Italy) under Contract PSN 83-085.

The authors are with the Dipartimento di Ingegneria Elettronica, Università di Firenze, Florence, Italy.
}

and therefore the earth stations concentrate the traffic from many other stations. For burst-type traffic, the TDMA structure does not achieve a high performance and efficiency. In these cases, the use of random-access techniques such as ALOHA [11] is a convenient approach, thus allowing each station to access the satellite directly.

In an SS/TOMA system, random-access techniques can be efficiently used in the uplink channels, while the TDMA techniques yield to the more suitable strategy in the downlink channels, where the traffic always presents a more continuous and regular flow.

The use of ALOHA techniques in multibeam packet satellites was studied by Chang [12]. This author has shown that a multibeam satellite system using an ALOHA protocol in the uplink channels gives a better performance with respect to a system using TDMA, if suitable protocols are employed.

In this paper, the performance of some models of a packet satellite communication system employing a random-access technique like slotted-ALOHA (S-ALOHA) for bursty traffic in the uplinks and a TDMA technique in the downlinks is proposed and analyzed. The theoretical performance of the models is studied and the results of extensive simulations are presented.

The first model considered assumes that:

1) the satellite has on-board processing capabilities and provides infinite buffers for successful packets;

2) the entire area covered by the satellite is divided into $N$ spatially disjoint spot beam zones and $N$ on-board transponders are available;

3) the earth stations of a beam zone share the common uplink channel using the well-known S-ALOHA protocol: the time axis is sliced into slots, each equal to the fixed duration of a packet;

4) the satellite processor determines whether overlaps occur by checking an error detection code; successful packets are demodulated to baseband, and their destination zone is derived;

5) in case the successful packets cannot receive immediate service, they are stored in the buffers assigned to the downlink zone and queued until a slot in the TDMA downlink channel is available;

6) the service provided by each transponder has its own frame structure, which is filled by transmitting packets (if any) from the corresponding buffer in a FIFO manner (First-In First-Out discipline); 
7) a fixed part of a TDMA frame, structured in minislots, is devoted to sending positive acknowledgments back to the earth stations of the beam zone; the minislots are organized in a fixed assignment TDMA;

8) the earth stations under a coverage area receive all the packets of their common zone: by inspecting the packet header at each terminal it is possible to determine the particular station the packet is destined to; packets addressed to other terminals are ignored.

The system performance will be theoretically analyzed by using the $M / G / 1$ queveing model, based on the appropriate evaluation of the service time distribution $(G)$, and extensive computer simulation results in close accordance with the theoretical analysis will also be reported.

An evolution of this model is further proposed: the number of transponders is reduced to $M<N$ in order to improve the average transponder utilization. A higher utilization is obtained at the expense of longer average delays. Full interconnection among earth stations is achieved by a channel-hopping capability of the transponders: a number of strategies of transmission are proposed, and their performance is compared.

The obtained results have shown that the first model $(N$ transponders) achieves small delays for the packets together with low transponder utilization. The second model $(M$ transponders) achieves higher transponder utilization, while increasing the packet time delays.

\section{Multibeam Satellite System with Uplink aloha and DOWNLINK TDMA PROTOCOLS}

In this section, the first model of a multibeam packet satellite system using random-access techniques in the uplink channels is described. In this model, the number of transponders on-board the satellite is assumed equal to the number of spot beams, so the model is called FTA (Fixed Transponder Assignment).

The general structure of the analyzed multibeam satellite system is shown in Fig. 1. $N$ different spot-beam antennas cover spatially disjoint areas and permit a complete interconnection between the earth stations. Each different area contains $N_{s}$ stations, which share a common transmission channel provided by the satellite spot beam.

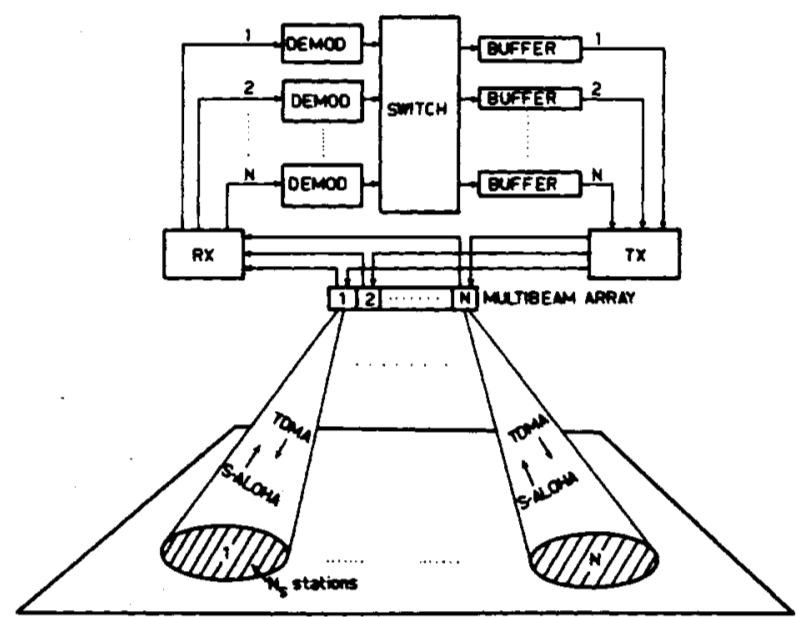

Fig. 1. General structure of the multibeam packet satellite system.
Earth stations transmit data messages in packets having length equal to $N_{b}$ bits, according to a slotted-ALOHA protocol. The overlapped packets are retransmitted randomly over a set of $K_{r}$ slots with a uniform distribution. The transmission rate is assumed equal to $v_{s}$ bits per second.

Successful packets are demodulated and stored in buffers on-board the satellite according to the destination zone. Every transponder is connected permanently to a particular spot beam and a different buffer is connected to each transponder.

The information is transmitted from the satellite to the earth stations according to a TDMA strategy, with the structure shown in Fig. 2. The TDMA periodic frame, having

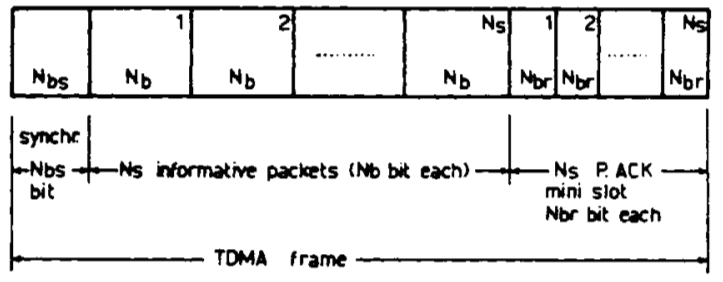

Fig. 2. General structure of the TDMA frame.

a time duration $T$, is divided into two parts. The first part is comprised of $N_{s}$ informative slots, each $\tau$ seconds long, equal to a packet duration, i.e.,

$$
\tau=N_{b} / v_{d}
$$

where $v_{d}$ is the downlink transmission rate. These slots are filled with packets (if any) from the corresponding buffer, according to a FIFO strategy.

The second part of the TDMA frame is comprised of some noninformative slots, subdivided as shown in Fig. 2. Specifically, we have $N_{s}$ minislots, each of $N_{b r}$ bits, used to transmit positive acknowledgments (P-ACK) to the earth stations, within the considered spot beam, that have successfully transmitted a packet in the previous time interval. In addition, at the beginning of the TDMA frame $N_{b s}$ bits are present for synchronization.

A TDMA frame therefore contains $N_{f}$ bits given by

$$
N_{f}=N_{s}\left(N_{b}+N_{b r}\right)+N_{b s} \text {. }
$$

$N_{f}$ is always assumed to be a multiple of $N_{b}$, which means

$$
N_{s} N_{b r}+N_{b s}=L N_{b}
$$

with $L$ a positive integer. Therefore, the frame duration is

$$
T=N_{f} / V_{d}=\tau\left(L+N_{s}\right) \text {. }
$$

\section{Theoretical analysis of the fTA Model}

In this section some performance parameters of the FTA model are theoretically derived. The analysis is carried out by means of the $M / G / 1$ queueing theory based on the embedded Markov chain method [13] and assumes the following two conditions:

i) the transmission delay $D_{t}$ is set equal to $0.25 \mathrm{~s}$;

ii) the packet generation at each earth station is modeled as a Poisson process with mean interarrival time $E_{i ;}$ of course, with the ALOHA protocol a station cannot generate a new packet when it is in the retransmission mode. 
In the system described in this paper the acknowledgments for the correctly received packets are sent to the earth stations in fixed parts of the downlink frame, according to a TDMA protocol; as a consequence, the time between a packet transmission and its acknowledgment is

$D_{\mathrm{ACK}}(t)=D_{t}+\frac{N_{b r}}{v_{d}}+t$,

where $t \in[0, T]$ is a random variable.

A station enters the retransmission state if no positive acknowledgment is received in the interval $D_{A C K}(T)$ after the transmission attempt. If we define the mean S-ALOHA packet delay, $d^{\prime}$, as the mean interval between the successful access on-board the satellite and the generation instant of a packet, we can put

$$
d^{\prime}=\tau+\frac{D_{t}}{2}+R_{t \times}\left[D_{\mathrm{ACK}}(\tau)+\left(\frac{K_{f}}{2}+1\right) \tau\right]
$$

where $R_{t x}$ is the mean number of packet retransmissions; $d^{\prime \prime}$ represents the mean time interval spent by a packet in the S-ALOHA uplink channel. The mean delay $d^{\prime}$ computed by (4) is reported in Fig. 3 as a function of $E_{t}$ (dotted curves).

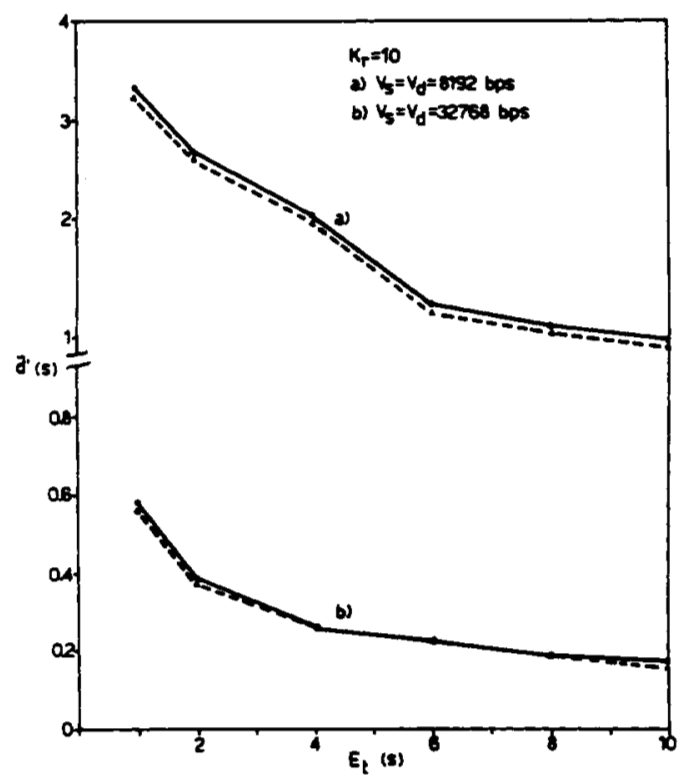

Fig. 3. Mean delay in S-ALOHA uplinks.

In the same figure, the value of $d^{\prime}$ obtained by computer simulations (continuous curves) is also shown. The results given by the theoretical analysis are quite similar to those obtained through the simulation.

Hypotheses i) and ii) have as a consequence that the mean effective interarrival time $E_{t}^{*}$ between successful packets of an earth station is increased with respect to the Poisson theoretical parameter $E_{t}$, because a station in the retransmission state cannot generate a new packet. The time spent by a station in the retransmission state depends on $E_{t}$ and also on $v_{s}$ and $v_{d}$. Indeed, by increasing $v_{s}$ the probability of packet overlapping decreases with $\tau$. Moreover, as $v_{d}$ increases, the mean time between retransmissions decreases. Therefore, the effective Poisson parameter $E_{t}^{*}$ is different from the theoretical $E_{t}$, and is a function of the form $E_{t}^{*}=f\left(E_{t}, v_{s}, v_{d}\right)$ :
Fig. 4 reports the functional dependence of $E_{t}^{*}$ on $E_{t}$ for different values of $v_{s}$ and $v_{d}$ and shows that $E_{t}^{*}$ is close to $E_{t}$ under low contention conditions.

By assuming that the packets generated by each earth station can be addressed, with the same probability, to any of the other $N N_{s}-1$ earth stations, we can suppose that

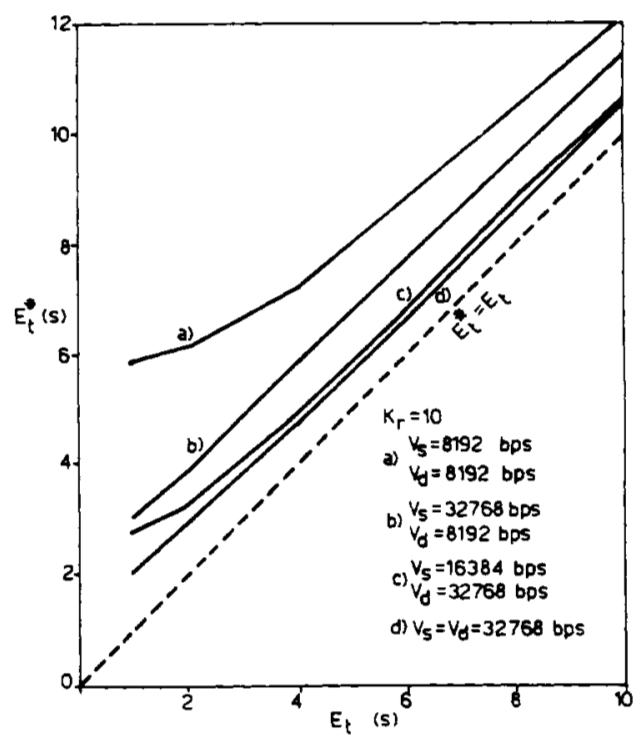

Fig. 4. Effective mean interarrival time as a function of the theoretical mean interarrival time.

the traffic at the input of each on-board buffer is still a Poisson process with rate $\lambda=\tau N_{s} / E_{t}^{*}$ packets/slot, as a function of $E_{t}, v_{s}$, and $v_{d}$. Therefore, the probability that $k$ packets arrive in a time slot $\tau$ at a given buffer is

$$
P(k)=\frac{\lambda^{k}}{k !} e^{-\lambda}
$$

The service time $(s t)$ for a packet is defined as the time interval between the first instant $k \tau$ ( $k$ integer) at which the top of the buffer queue is reached and the time of its departure from the buffer. The service time is a random variable, because of the presence of the synchronization bits and the P-ACK slots. Indeed, the time $T$ of a TDMA frame is not completely devoted to the transmission of informative packets. The service is periodically interrupted for the transmission of the synchronization bits and the positive acknowledgments (Fig. 2). Therefore, in the analysis of the service time, these interruptions (service rest periods) must be taken into account.

Moreover, it should be noted that the service time for a packet depends on the buffer state, which can be busy when other packets, previously arrived, are present in the buffer or idle when no packet is waiting for service in the buffer.

The busy state is considered first. The present packet $C_{n+1}$ obtains service only when the previous packet $C_{n}$ in the buffer queue leaves the system. Two different cases can occur, depending on the time instant at which the service of $C_{n}$ ends. The first case (Fig. 5(a)) occurs when the $C_{n}$ service ends before the $\left(N_{s}-1\right)$ th informative slot $(l)$ of the TDMA frame and therefore $C_{n+1}$ can receive immediate service. The service time is therefore one time slot.

By denoting with $P_{s t}(i \mid X)$ the probability of a service time equal to $i$ time slots given the buffer in the state $X(X=B$ 
(a)

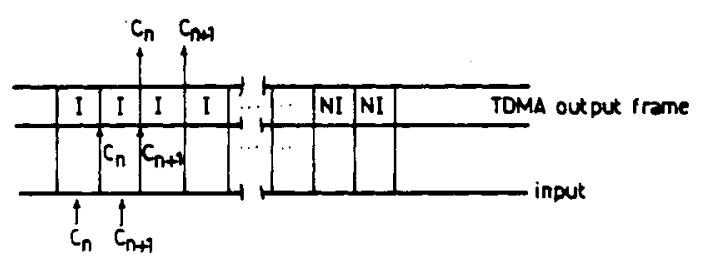

(b)

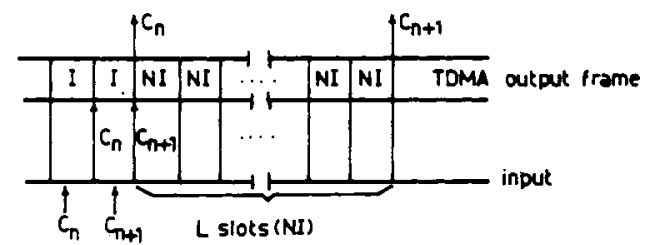

Fig. 5. Busy period service conditions. (a) Immediate service for $C_{n+1}$. (b) Nonimmediate service for $C_{n+1}$.

for the busy state and $x=l$ for the idle state), we have in this case

$$
P_{s t}(1 \mid B)=\frac{N_{s}-1}{N_{s}}
$$

When the $C_{n}$ service ends in the last informative time slot of the TDMA frame, $C_{n+1}$ cannot be served until the beginning of the informative slots of the successive frame. Indeed, the following $L$ time slots are reserved to noninformative (NI) transmissions (Fig. 5(b)). Therefore, the service time is $(L+1)$ time slots and it results in

$$
P_{s t}(L+1 \mid B)=\frac{1}{N_{s}} \text {. }
$$

Even in the case of an idle system, two different situations can occur, depending on the instant when the packet

the system during the time interval (between the arrival and the departure) of $C_{n}$, when the system is in the idle state, and $\Delta q_{n}$ is equal to 1 if $q_{n} \geqslant 1$ and to 0 if $q_{n} \leqslant 0$.

The analysis of the system can be carried out by considering the behavior of the system at the instants of the packet departures. Following the analysis of the embedded Markov chain given in [13]-[18], (10) can be subdivided in two different cases depending on the value of $\Delta q_{n}$

$$
\begin{array}{ll}
q_{n+1}=q_{n}-1+v_{n+1}, & \text { if } \Delta q_{n}=1(\text { case B) } \\
q_{n+1}=w_{n+1}, & \text { if } \Delta q_{n}=0(\text { case } 1) .
\end{array}
$$

As is shown in the Appendix, the z-transform $Q(z)$ of the probability distribution of the queue lengths $q_{n}$ is

$$
Q(z)=p_{0} \frac{z W(z)-V(z)}{z-V(z)}
$$

where $p_{0}$ is the probability of an idle buffer $\left(q_{n}=0\right)$, while $V(z)$ and $W(z)$ are the $z$-transforms of the probability distribution of the number of customer arrivals $v_{n}$ and $w_{n}$, respectively.

By recalling that the arrivals are a Poisson process, it can be shown that (see the Appendix)

$$
\begin{aligned}
V(z)= & \frac{N_{s}-1}{N_{s}} e^{\lambda(z-1)}+\frac{1}{N_{s}} e^{\lambda(L+1)(z-1)} \\
W(z)= & \frac{e^{-\lambda}\left(e^{\lambda z}-1\right)}{z\left(1-e^{-\lambda}\right)} \\
& \cdot\left[\frac{N_{s}}{N_{s}+L} e^{\lambda(z-1)}+\frac{1}{N_{s}+L} \sum_{n=2}^{L+1} e^{n \lambda(z-1)}\right] .
\end{aligned}
$$

By setting $z=1$ in (13), then $Q(1)=1$ and $p_{0}$ is given by

$$
p_{0}=\frac{\left[N_{s}-\left(N_{s}+L\right) \lambda\right]\left(1-e^{-\lambda}\right) / N_{s}}{\lambda\left[\frac{1}{N_{s}+L}\left(2 N_{s}+\frac{(L+2)(L+3)-6}{2}\right)-\frac{e^{-\lambda}}{N_{s}+L}\left(N_{s}+\frac{2 L+L(L+1)}{2}\right)-\frac{N_{s}+L}{N_{s}}\left(1-e^{-\lambda}\right)\right]} .
$$

$C_{n+1}$ arrives. If $C_{n+1}$ arrives before the beginning of the last informative time slot of the TDMA frame (Fig. $6(\mathrm{a})$ ), it can receive immediate service and therefore we have

$$
P_{s t}(1 \mid I)=\frac{N_{s}}{N_{s}+L} \text {. }
$$

On the other hand, when a packet is ready for service in correspondence with noninformative slots, it can receive service only in the first informative time slot of the successive frame (Fig. 6(b)). The service time duration depends on the actual arrival slot. The probability of a service time equal to $k$ slots is then

$$
P_{s t}(k \mid l)=\frac{1}{N_{s}+L}, \quad k=2,3, \cdots, L+1 .
$$

The state equation for the system previously described can be written as

$$
q_{n+1}=\left(q_{n}-1+v_{n+1}\right) \Delta q_{n}+w_{n+1}\left(1-\Delta q_{n}\right)
$$

where $q_{n}$ denotes the queue length after the departure of the $n$th packet, $v_{n}$ denotes the number of packets arriving during the service time of the $n$th packet, when the system is in the busy state, $w_{n}$ is the number of packets entering
By definition we obtain the mean queue length $\bar{q}$

$$
\begin{aligned}
\bar{q}= & \left.\frac{d Q(z)}{d z}\right|_{z=1}=\frac{1}{2\left(1-\frac{N_{s}+L}{N_{s}} \lambda\right)} \\
& \cdot\left(p_{0} \frac{\lambda^{2}}{\left(N_{s}+L\right)\left(1-e^{-\lambda}\right)}\right. \\
& \cdot\left\{4 N_{s}+\frac{(L+2)(L+3)(2 L+5)-30}{6}\right. \\
& \left.-\left[N_{s}+\frac{(L+1)(L+2)(2 L+3)-6}{2}\right] e^{-\lambda}\right\} \\
& \left.-\frac{p_{0} \lambda^{2}}{N_{s}}\left[N_{s}+L(L+2)\right]+\frac{\lambda^{2}}{N_{s}}\left[N_{s}+L(L+2)\right]\right)
\end{aligned}
$$

Relations (15) and (16) will be compared with the simulation results in Section $V$.

Applying Little's formula $\bar{q}=\lambda D_{b}$, from (16) we easily obtain the average time $D_{b}$ spent waiting in the buffer queue by a packet. The average service time $D_{s}$ can be evaluated as 


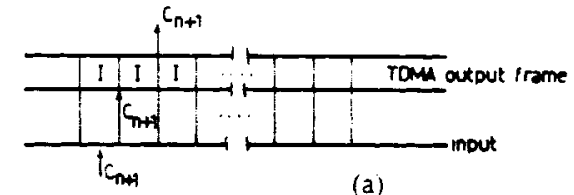

(a)

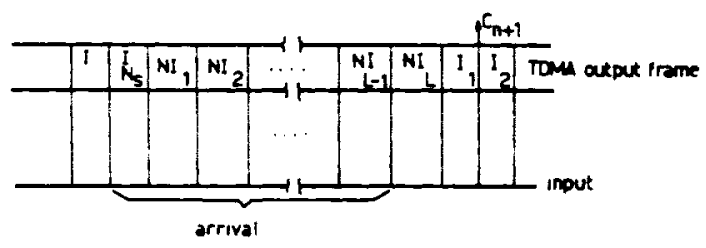

(b)

Fig. 6. Idle period service conditions. (a) Immediate service for $C_{n+1}$. (b) Nonimmediate service for $C_{n+1}$.

$$
\begin{aligned}
D_{s}= & {\left[1 \cdot \frac{N_{s}-1}{N_{s}}+(L+1) \frac{1}{N_{s}}\right]\left(1-p_{0}\right) } \\
& +\left(1 \cdot \frac{N_{s}}{N_{s}+L}+\sum_{k=2}^{L+1} k \frac{1}{N_{s}+L}\right) p_{0} \\
= & \frac{N_{s}+L}{N_{s}}\left(1-p_{0}\right)+\frac{2 N_{s}+L^{2}+3 L}{2\left(N_{s}+L\right)} p_{0} .
\end{aligned}
$$

The total time spent in the system (queue plus service) is $D_{b}+D_{s}$ and the total number of customers in the system is $\lambda\left(D_{b}+D_{s}\right)$. The global end-to-end mean packet delay is therefore

$$
\bar{D}=D_{b}+D_{s}+d^{\prime}+\frac{D_{t}}{2}
$$

\section{IV. a Multibeam Satellite System with a Reduced NUMBER OF TRANSPONDERS}

As is well known, the average utilization of a communication channel using a slotted-ALOHA protocol is at most 36.8 percent [11]-[19]. By assuming a uniform distribution of the packets among all the stations, the informative flow that comes out from each buffer in the previously discussed multibeam satellite model has similar characteristics. In this way, the average utilization of each transponder, taking into account both the informative flow and the service traffic (synchronization and positive acknowledgments), achieves typical values of about 30 percent.

In this section some multibeam satellite techniques are described which use a number $M$ of transponders lower than the number $N$ of the spot beams, in order to increase the transponder utilization. In these techniques, the transponders are dynamically connected to the spot beams according to a predetermined switching strategy, which is always the same for each transponder. This approach significantly reduces the complexity of the implementation of the switching algorithm with respect to similar strategies.

A common feature of all the following models is that of having the frame duration $T$ subdivided into intervals, each $(P+\Delta)$ seconds long, $P$ and $\Delta$ being two given real numbers, as shown in Fig. 7. The subintervals $P$ are reserved for the transmission of informative packets and the subintervals $\Delta$ to the transmission of positive acknowledgments. During the subintervals $\Delta$, the satellite processor recognizes the

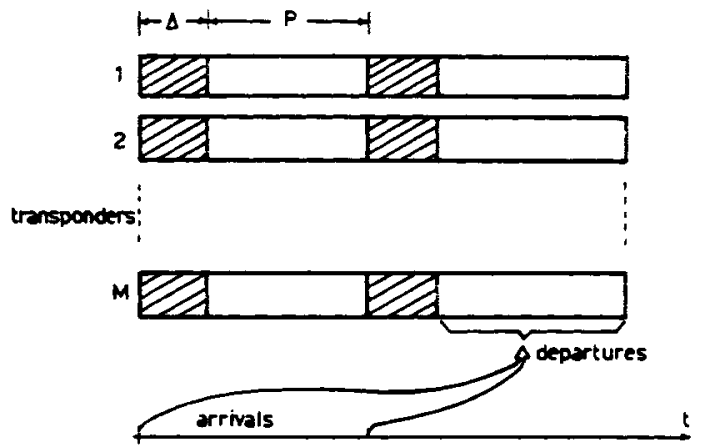

Fig. 7. Allocation of the TDMA frame in the model with a reduced number of transponders ( $\Delta$ : noninformative frame part; $P$ : informative frame part).

addresses of buffered packets and chooses the appropriate switching strategy of the transponders over the downlink beams. We assume that two transponders are not allowed to be connected to the same beam in the same time slot. Moreover, the switching time is assumed as negligible. When a packet arrives on-board the satellite in a given interval $(P+\Delta)$, its transmission is delayed to the next interval $(P+\Delta)$, even if the system is in the idle state.

The general structure of the on-board equipment is shown in Fig. 8, where MS denotes the switching matrix. A com-

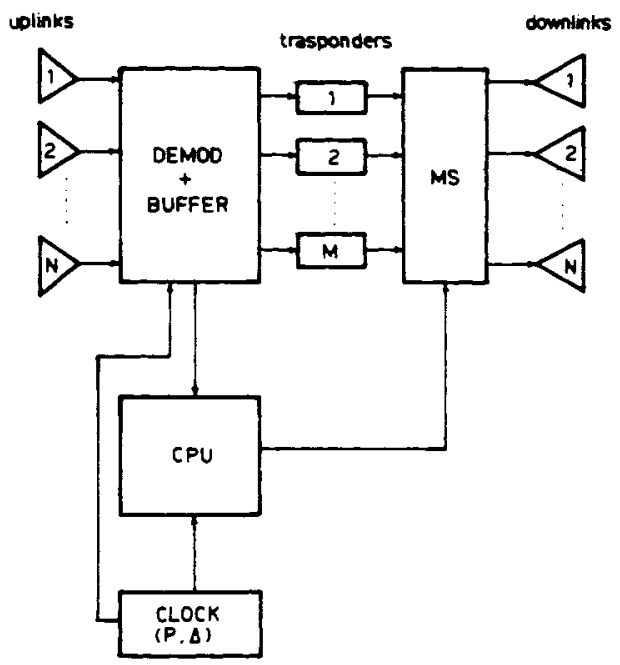

Fig. 8. General block diagram for the model with a reduced number of transponders (MS: Switching Matrix).

mon buffer is present in the system, in which all the successfully received packets are stored.

In the following some strategies are described which differ for the number of transponders and the distribution of the intervals $P$ and $\Delta$ in the TDMA frame.

\section{A. DS1 Strategy}

The first Dynamic-Switching strategy (denoted in the following as DS1) refers to a number $M$ of transponders equal to $N / 2$. The general structure of the frames in the $M$ downlinks is shown in Fig. 9. The ith transponder $(1 \leqslant i$ $\leqslant \mathcal{M})$ is connected in each TDMA frame to the $i$ th and $[(N / 2)+i)]$ th downlink beams. 


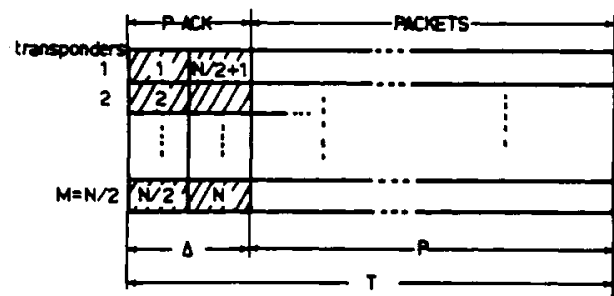

Fig. 9. TDMA frames structure for DS1 model.

The mean time $D_{m}$ a packet is delayed due to the realization of the switching strategy is $T / 2$, where $T=P+$ $\Delta$. This delay must be considered in the total mean packet delay.

\section{B. DSSki Strategies}

In order to reduce $D_{m}$ we have considered a class of more general strategies in which the switching operation is more frequently activated. The number of transponders is $M=N / k, k$ being an integer (and, of course, a submultiple of $N$ ) and the frame $T$ is subdivided into $i$ subintervals, each $(P+\Delta)$ seconds long. These strategies are denoted in the following as DSSki and the general frame structure is shown in Fig. 10.

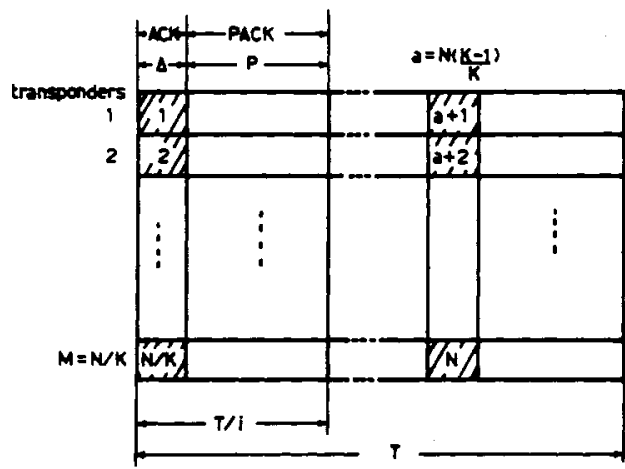

Fig. 10. TDMA frames structure for DSSki models.

The switching operation, implemented during the noninformative subintervals $\Delta$, is activated $i$ times in each frame and so the mean delay $D_{m}$ can be reduced by suitably choosing the parameters of the system. On the other hand, if the transponders are switched among the beams too frequently, the number of time slots in a frame which are utilized for noninformative transmissions (subintervals $\Delta$ ) increases and therefore the number of slots which can be utilized for the transmission of informative packets is reduced. The same effect arises when the number of transponders is decreased. For a heavy traffic and particular choices of the system parameters, the on-board queue can significantly increase and the system can become unstable. However, if the parameters of the system are correctly chosen, the utilization of the transponders can be significantly improved without highly increasing the mean delay time.

In particular, three different strategies, the frame structures of which are depicted in Fig. 11, are analyzed. The first strategy (Fig. 11(a)) is of type DSS22 and has the following parameters: $M=N / 2, T=18 \tau, N_{b}=1024, \Delta=2 \tau, P=7 \tau$. (a)

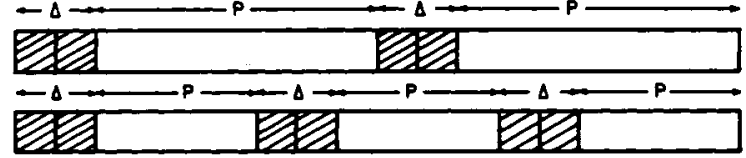

(c)

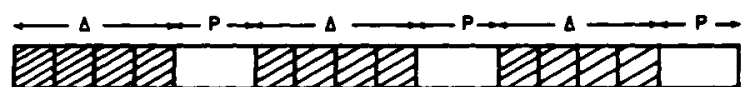

Fig. 11. TDMA frames structure for the models. (a) DSS22. (b) DSS33. (c) DSS63.

This strategy was considered an alternative to DS1 in order to show the advantage of a more frequent switching operation. The second strategy (Fig. 11(b)) is of type DSS33, for which $M=N / 3, T=18 \tau, N_{b}=1024, \Delta=2 \tau, P=4 \tau$. The third strategy (Fig. 11(c)) is of type DSS63, for which $M=$ $N / 6, T=18 \tau, N_{b}=1024, \Delta=4 \tau, P=2 \tau$. This last strategy is a typical example where the noninformative part is predominant and therefore the system can easily become unstable.

\section{COMPARISON OF RESULTS}

In this section theoretical and simulation results for the two different classes of satellite communication models are presented. The overall performance of the systems is characterized by means of the following main parameters:

1) mean transmission delay per packet $(\bar{D})$, i.e., the mean time between the first ALOHA attempt at transmission and the successful arrival to the destination station;

2) mean transponder utilization factor $\left(U_{t}\right)$, defined as the ratio between the time spent in transmission and the total time;

3) mean interarrival time $E_{t}$.

Computer simulations have been performed by fixing the parameters $N=10, N_{s}=16, N_{b}=1024$. Accordingly, the downlink TDMA frame is composed of 18 slots: 16 informative slots (1024 bits each), 16 minislots (120 bits each), and 128 bits for synchronization.

For the first class, the FTA system, we also present the on-board queuing parameters $\bar{q}$ and $p_{0}$, obtained by simulation and by means of the theoretical relations developed in Section III.

As far as the influence of the uplink S-ALOHA protocol on the overall performance of the system is concerned, a decrease of $v_{s}$ (and therefore an increase of the slot duration $\tau$ ), with the other parameters held constant, increases the probability of the overlapping packets, and thus the mean number of retransmissions per packet $R_{t x}$ and the mean delay $\bar{D}$ get larger. These considerations agree with the behavior of Fig. 12 where the mean delay $\bar{D}$ is plotted, for different values of $v_{s}$, versus the mean theoretical interarrival time $E_{t} . K_{r}$ is another critical parameter of the S-ALOHA contention: by decreasing $K_{r}$, the mean number of retransmissions $R_{t x}$ and the mean delay $\bar{D}$ increase: Figs. 13 and 14 plot the dependence of the mean retransmission number $R_{t x}$ and the mean delay $\bar{D}$ on the parameter $K_{r}$ for three different values $\left(K_{r}=3,5,10\right)$.

By increasing the packet contention in the S-ALOHA channel, the throughput of the successful packets reaching 


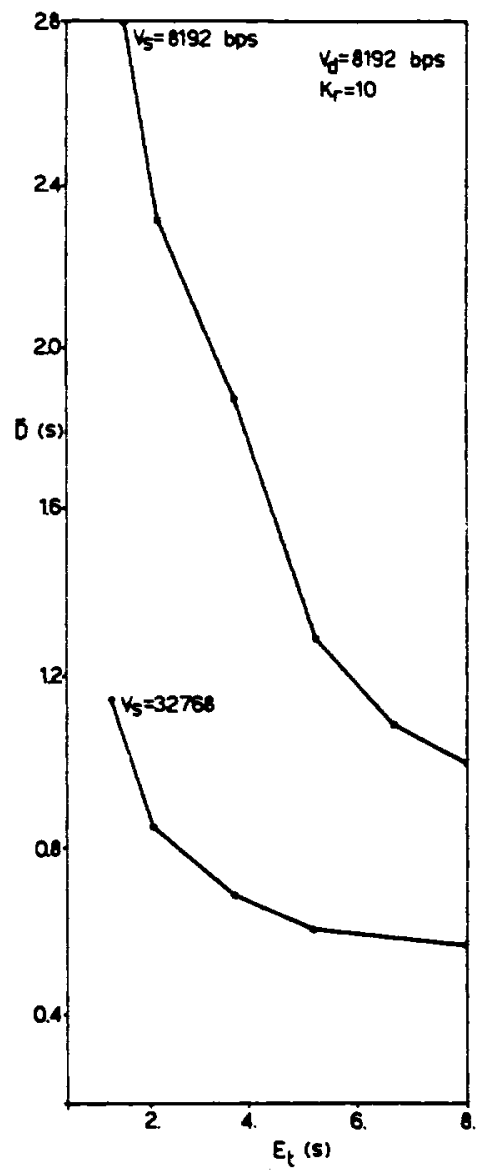

Fig. 12. FTA system: mean delay $\bar{D}$ versus $E_{t}$ for different values of $V_{s}$.

the satellite buffer decreases; consequently, the mean transponder utilization becomes lower. In Fig. 15, the different utilization factor $U_{t}$ given in percent values versus $E_{t}$ for three values of $K_{r}$ is shown.

The downlink transmission rate $v_{d}$ also influences the

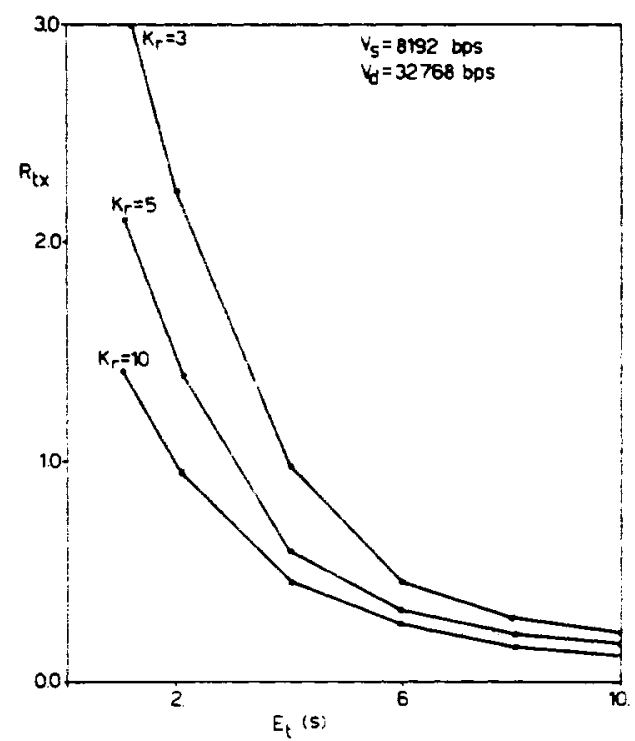

Fig. 13. FTA system: mean number of retransmissions $R_{t x}$ versus $E_{t}$ for different values of the retransmission interval $\kappa$.

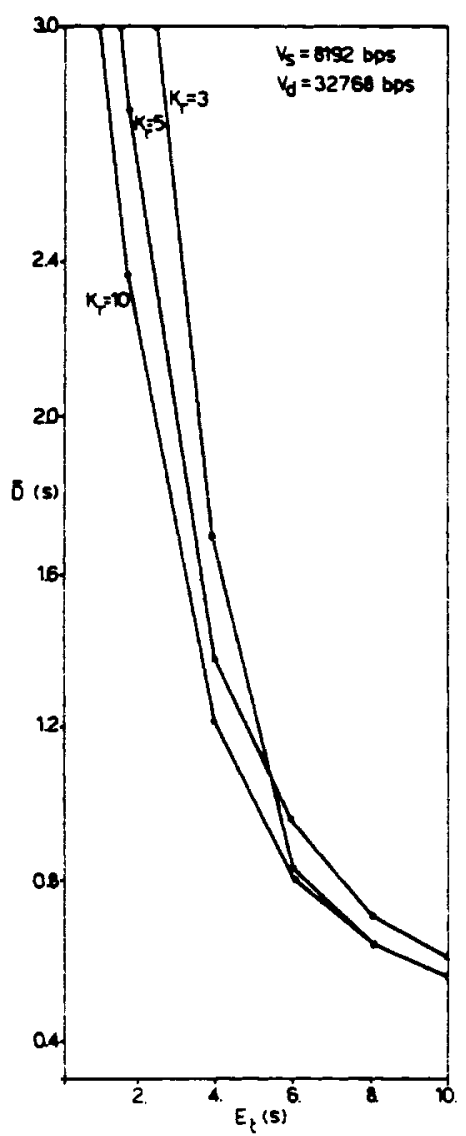

Fig. 14. FTA system: mean delay $\bar{D}$ versus $E_{t}$ for different values of $K_{r}$.

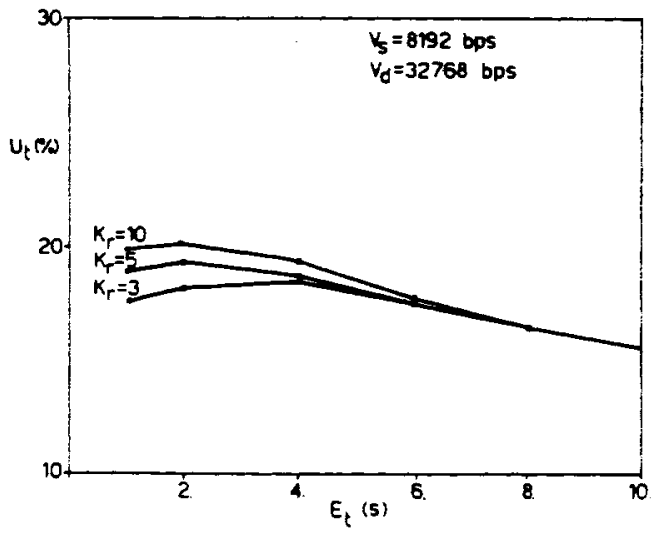

Fig. 15. FTA system: mean transponder utilization factor $U$ (percent) versus $E_{f}$ for different values of $K_{r}$.

mean delay $\bar{D}$. As previously observed, the mean interval required to receive positive acknowledgments at the earth stations grows as $v_{d}$ decreases and this influences the effective interarrival time between packets, i.e., the effective mean channel input rate. These considerations are confirmed by Fig. 16 where $\bar{D}$ is plotted versus $E_{t}$ for two values of $v_{d}$.

As a concluding remark, we note that in the FTA system, a low transmission delay $\bar{D}$ can be obtained bv means of a low contention condition on the S-ALOHA channels (i.e., high $v_{s}$ and $E_{t}$ and appropriate values of $K_{r}$ ). 


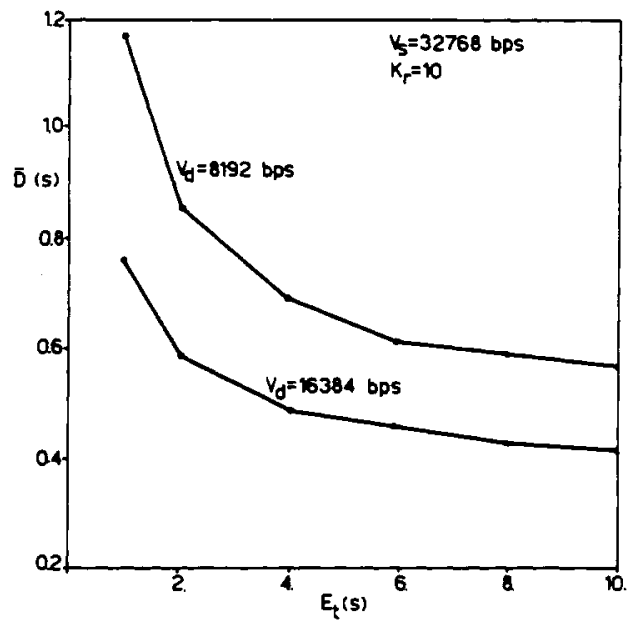

Fig. 16. FTA system: mean delay $\bar{D}$ versus $E_{t}$ for different values of the downlink rate $v_{d}$.

Let us now examine the performance of the on-board queue system. By increasing $v_{s}$, thus reducing the S-ALOHA contention, the number of on-board successful arrivals grows and so does the mean queue length $\bar{q}$. In Fig. 17, the parameter $\bar{q}$ is plotted as a function of traffic $\left(E_{t}\right)$ for two

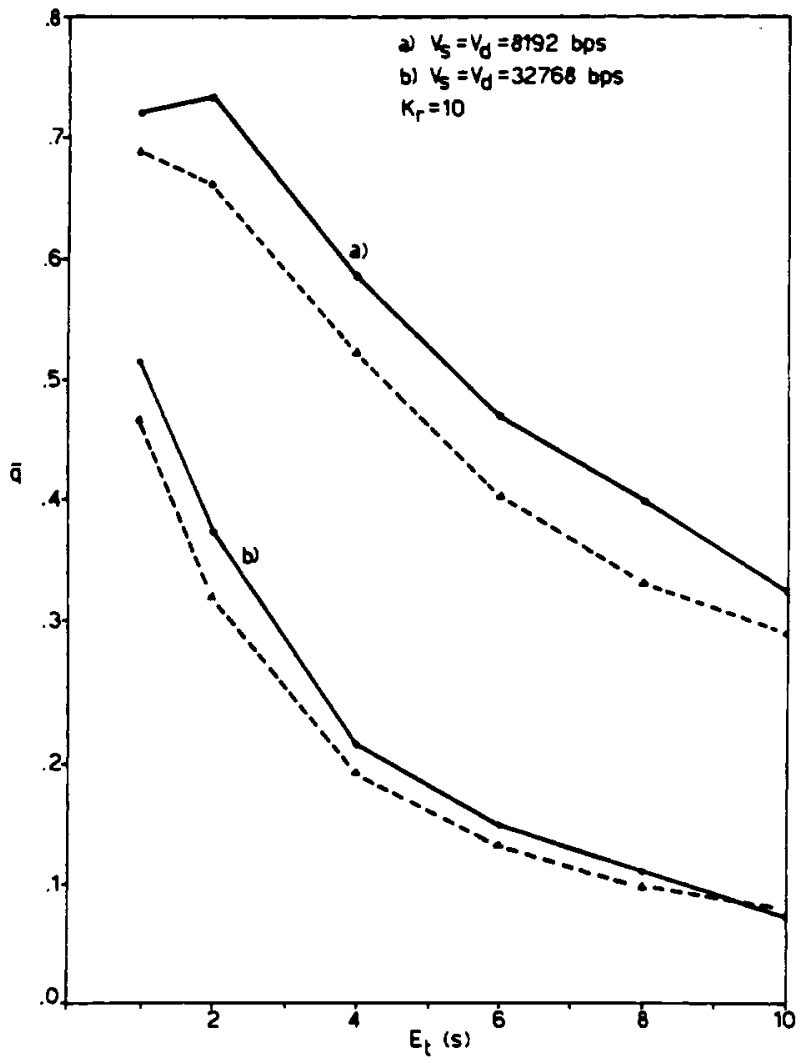

Fig. 17. FTA system: mean queve length $\bar{q}$ versus $E_{t}$ for two different values of the uplink rate $v_{s}$ (continuous line: simulation results; dashed line: theoretical results).

values of $v_{s}$. The continuous lines represent simulation data, and the dotted lines indicate queueing theory predictions. Fig. 18 shows the probability of an empty buffer $\left(p_{0}\right)$ for the same values of the system parameters $v_{s}, v_{d}, K_{r}$, and $E_{t}$.

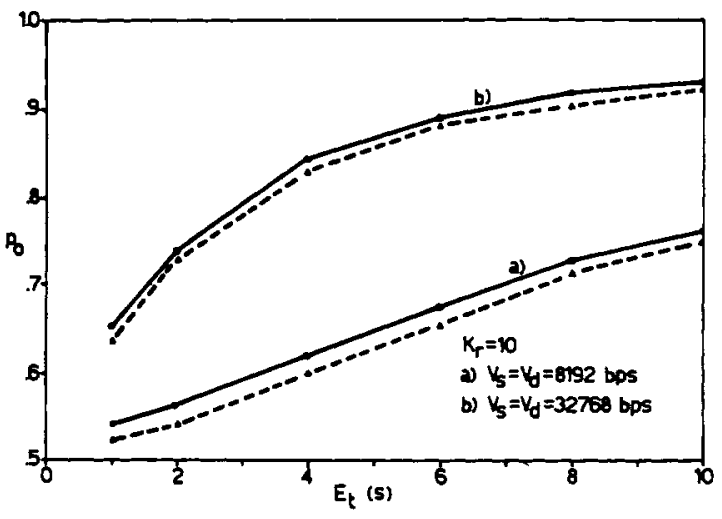

Fig. 18. FTA system: probability of an empty buffer $p_{0}$ versus $E_{t}$ for two different values of $v_{s}$ (continuous line: simulation results; dashed line: theoretical results).

The figures show a satisfactory agreement between the theoretical and the simulation values. This result justifies a posteriori the assumption of a Poisson process for the mean input rate $\lambda$.

Good global end-to-end performance is obtained by employing high $v_{s}$ and $v_{d}$ i however, for a fixed traffic intensity, the reduction of downlink slot duration decreases the mean transponder utilization, as can be seen from Fig. 19(a) and (b). The previous results show that the FTA strategy achieves a good performance from the user's point of view (end-to-

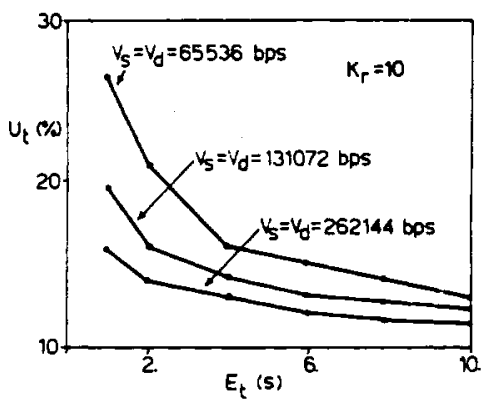

(a)

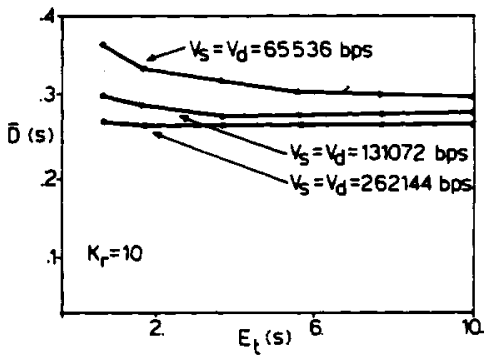

(b)

Fig. 19. FTA system performance. (a) Mean transponder utilization factor $U_{t}$ (percent) versus $E_{t}$ for different values of transmission rate. (b) Mean delay $\bar{D}$ versus $E_{t}$ for different values of transmission rate.

end delay) at the expense of a relatively low satellite system performance (low transponder utilization).

Let us now turn to consider the performance of the second class of strategies having a number of transponders lower than the number of spot beams. In Figs. 20 and 21, the mean delay $\bar{D}$ and the utilization $U_{t}$ are presented for all strategies as a function of $E_{t}$ for fixed values of the systems parameters. In these figures and in the following 


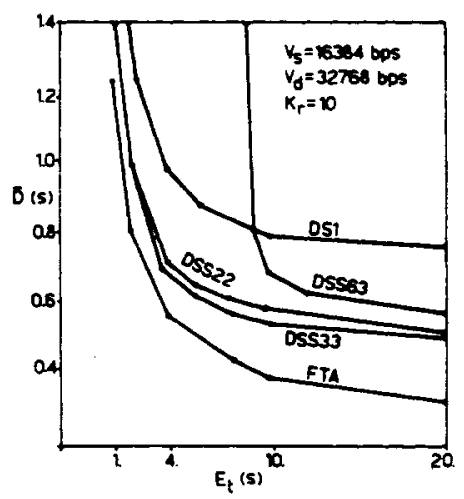

Fig. 20. Mean delay $\bar{D}$ versus $E_{t}$ for the different transmission models.

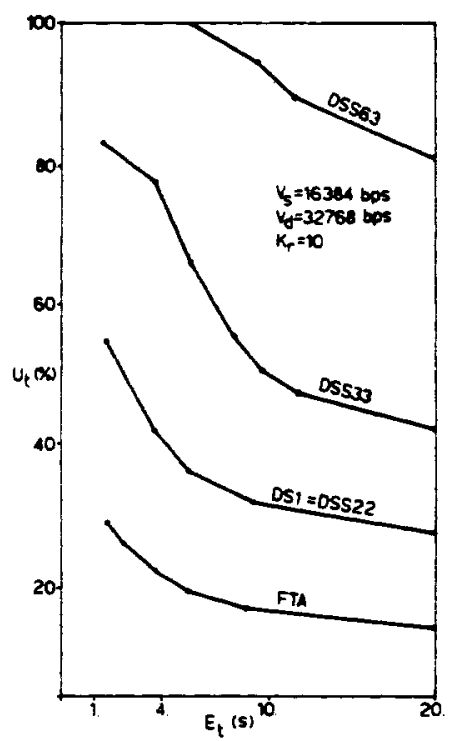

Fig. 27. Mean utilization factor $U$,(percent) versus $E_{t}$ for the different transmission models.

ones we also present, for comparison, the results of the FTA model. We note that the mean delay obtained by DSS 22 is lower than that obtained by DS1. These results are related to a more frequent switching operation on-board the satellite. The transponder utilization is the same for the two models, since the transponder number is equal. For these reasons, the DS1 strategy will be no longer presented in the following. DSS33 is characterized by a lower $P+\Delta$ interval $(P+\Delta=T / 3)$ and a lower number of transponders with respect to DSS22; these characteristics explain, respectively, the lower mean delay and the higher transponder utilization for fixed traffic conditions.

The further reduction of the transponder number in DSS63 determines a corresponding decrease of the informative slots in the TDMA frame; this explains the higher mean delay of this model. The utilization factor grows due to the presence of a higher fraction of noninformative slots. Consequently, as the traffic increases, DSS63 can easily become unstable.

In order to characterize the critical behavior of the DSSki systems, we define the minimum interarrival time $E_{t e}$ for each earth station as that particular mean interarrival time for which a number of packets equal to the total number of time slots available in all the transponders during the interval $P$, is successfully received on-board the satellite.
The mean number $n_{p}$ of packets successfully received at the satellite, in an interval $(P+\Delta)$ seconds long, is

$$
n_{\rho}=N_{s} N(P+\Delta) / E_{t}
$$

The buffer queve remains in stationary conditions if

$$
n_{p} \leqslant M v_{d} P / N_{b} \text {. }
$$

The value $E_{t c}$ for which the equality in (20) holds, is the minimum interarrival time

$$
E_{t \ell}=N_{s} \frac{N}{M} \frac{(P+\Delta)}{P} \frac{1}{v_{d}} .
$$

It depends on the choice of the model $(N / M, P, \Delta)$ and the system $\left(N_{s}, v_{d}\right)$ parameters.

Fig. 22 plots $E_{t l}$ versus $v_{d}$. For a fixed $v_{d}$, the maximum capacity of handling traffic increases ( $E_{t \ell}$ decreases) when the transponder number grows.

These considerations are in accordance with the behavior

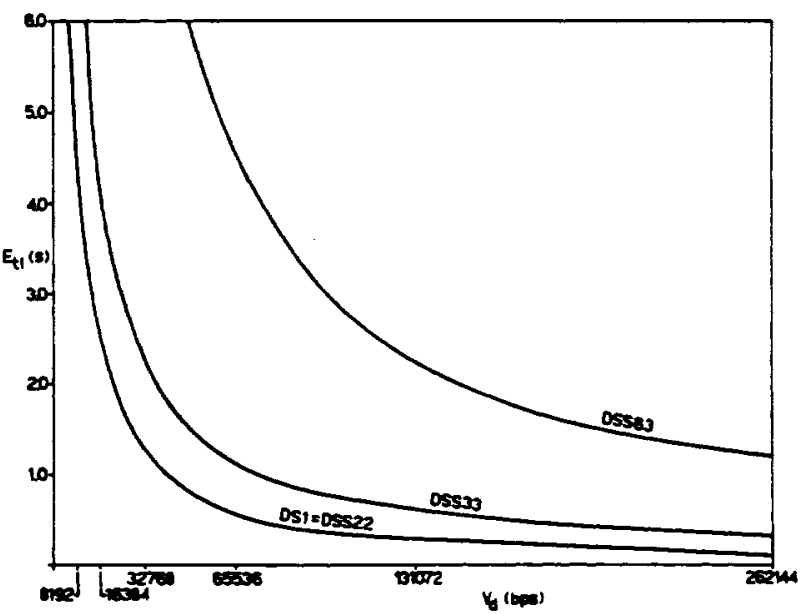

Fig. 22. Minimum interarrival time $E_{t}$ versus $v_{d}$ for the different transmission models.

of Figs. 23 and 24 . It can be noted that the stability bound $E_{t e}$ given in (21) is greater for DSS63 having a lower $v_{d}$. For the case shown in Fig. 23(b), the parameter $E_{t \ell}$ assumes a value of $4.5 \mathrm{~s}$, while in Fig. 23(a) it has the value of $1.125 \mathrm{~s}$. From Fig. 24(a) and (b) we can observe that the full transponder utilization (100 percent) corresponds to unstable conditions, as the excess of the queue length would permit to fill the TDMA downlink frame completely.

\section{CONCLUSIONS}

In this paper we have described and analyzed multibeam packet satellite communication systems using the S-ALOHA protocol in the uplinks and TDMA techniques in the downlinks. Future satellites having on-board processing capabilities may conveniently employ different multiple-access techniques in the uplinks and the downlinks. Randomaccess techniques, such as S-ALOHA, in particular, allow each earth station to directly access the satellite transponders and are appropriate for the transmission of bursttype data traffic. The satellite processing capability also permits to detect packet collisions on-board, blocking the downlink transmission of unsuccessful packets to the earth receiving stations, and allows the earth sending stations to receive faster positive-acknowledgment replies for success- 


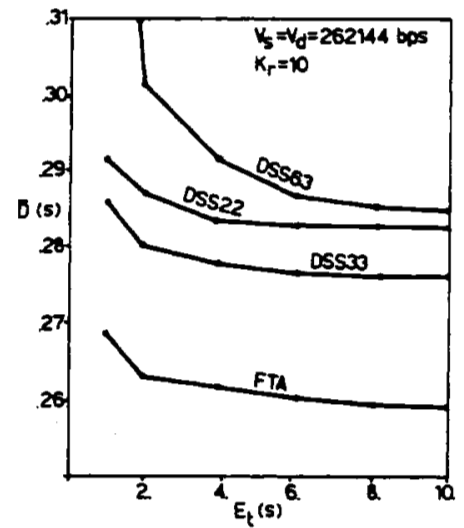

(a)

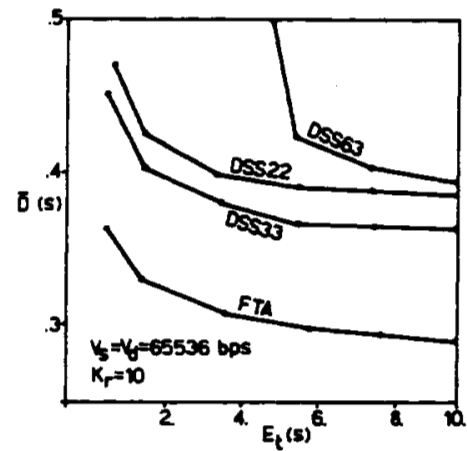

(b)

Fig. 23. Mean delay $\bar{D}$ versus $\boldsymbol{E}_{t}$ for the different transmissions models. (a) $v_{s}=v_{d}=262144$ bits $/ \mathrm{s}$. (b) $v_{s}=v_{d}=$ 65536 bits $/ \mathrm{s}$.

ful packets. Through the on-board demodulation and detection processes, the address of the destination station for each packet is determined. Thus a convenient solution for the downlink protocol appears to be the TDMA technique, which avoids any packet collision and can achieve a higher system utilization.

The first system model analyzed in this paper employs a number of satellite transponders equal to the number of spot beams. In this case, a good end-to-end system performance is achieved at the expense of a relatively low transponder utilization. The second class of system models proposed and described has a reduced number of transponders compared to the number of spot beams. By a suitable choice of the relevant system parameters, a higher transponder utilization can be obtained together with a small increase in the end-to-end mean packet delay. In this case, however, the parameter choice is critical: unstable conditions can occur with a careless system design.

In addition to these considerations, it should be noted that even the system reliability design can take advantage of the results presented in this paper. A satellite system, having a number of transponders equal to the number of spot beams, in the presence of faulty transponders, can be reconfigured in order to employ a lower number of transponders switched among all the spot beams with a timesharing philosophy. The transponder reconfiguration is still able to assure a good global performance, given an appropriate design of the system parameters.

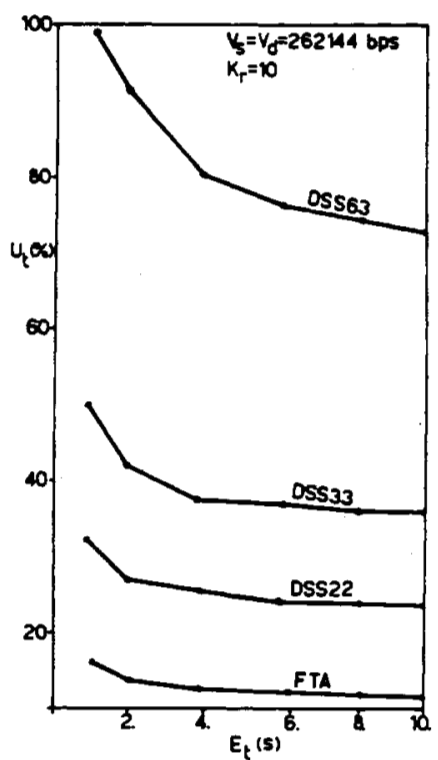

(a)

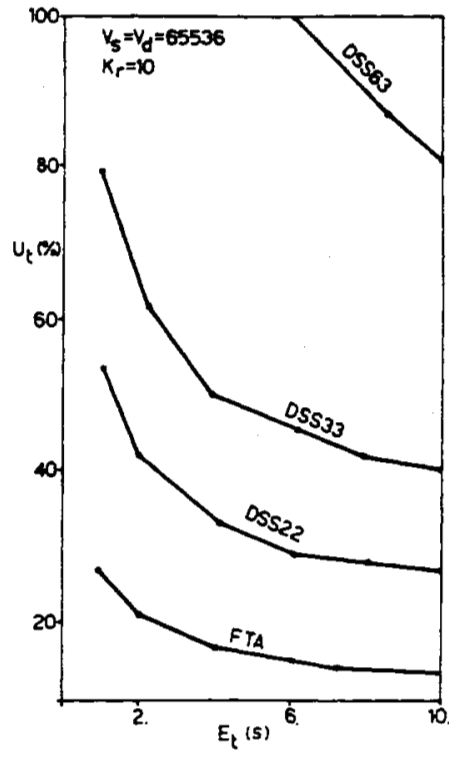

(b)

Fig. 24. Mean utilization factor $U_{t}$ (percent) versus $E_{t}$ for the different transmission models. (a) $v_{s}=v_{d}=262144$ bits $/ \mathrm{s}$. (b) $v_{s}=v_{d}=65536 \mathrm{bits} / \mathrm{s}$.

\section{APPENDIX}

In this Appendix we derive (13) and (14) of the text.

We recall that $v_{n}$ is the number of packets arriving during the service time of the $C_{n}$, when the system is in the busy state, and $w_{n}$ is the number of packets entering the system during the time interval between the arrival and the departure instants of $C_{n}$, when the system is in the idle state. The system state equations are given by (10)-(12) of the text. The following derivation is based on that outlined in [16], modified to take into account the different service time distribution we have for the proposed system. For simplicity, we drop the subscripts from $v_{n}$ and $w_{n}$, assuming a steady-state condition. 
The $z$-transform of the probability distribution $P(v=k)$ is given by

$$
V(z)=\sum_{k=0}^{\infty} P(v=k) z^{k}
$$

The probability of $k$ arrivals during the service time in the busy state is

$$
P(v=k)=\sum_{n=1}^{\infty} \frac{(n \lambda)^{k}}{k !} e^{-n \lambda} P_{s t}(n \mid B) .
$$

Using the values of $P(n \mid B)$ given in (6) and (7) we obtain (14a) of the text.

In the idle state case, at the departure instant, the first customer $C_{n}$ of an idle period leaves behind $w=w_{1}+w_{2}$ customers to be served (Fig. 25), where $w_{1}$ is the number of arrivals during the full slots of the service period and $w_{2}$ is

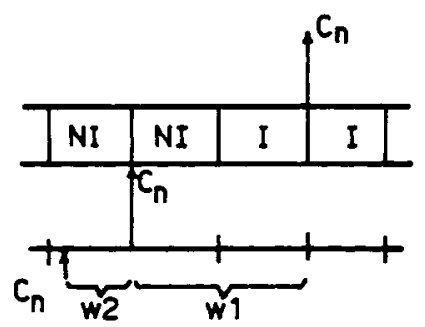

Fig. 25. Idle period: customer arrivals during the service period of $C_{n}$

the number of arrivals during the fractional slot beginning with the arrival of the customer. Because of the assumption of Poisson arrivals, $w_{1}$ and $w_{2}$ are independent. We have for $W_{1}(z)$, using $(8)$ and $(9)$

$$
\begin{aligned}
W_{1}(z) & =\sum_{k=0}^{\infty}\left[\sum_{n=1}^{\infty} \frac{(n \lambda)^{k}}{k !} e^{-n \lambda} P_{s t}(n \mid l)\right] z^{k} \\
& =\frac{N_{s}}{N_{s}+L} e^{\lambda(z-1)}+\frac{1}{N_{s}+L} \sum_{n=2}^{l+1} e^{n \lambda(z-1)} .
\end{aligned}
$$

As shown is [16], we have

$$
W_{2}(z)=\frac{e^{-\lambda}\left(e^{\lambda z}-1\right)}{\left(1-e^{-\lambda}\right) z}
$$

By the independence assumption, we obtain $W(z)$ as

$$
W(z)=W_{1}(z) W_{2}(z)
$$

Substituting (A3) and (A4) into (A5), we get (14b) of the text. Now we can derive (13). Defining

$$
Q_{n}(z)=\sum_{k=0}^{\infty} P\left(q_{n}=k\right) z^{k}
$$

we have, as shown in [15],

$$
Q_{n+1}(z)=\frac{Q_{n}(z) V(z)+p_{0}[z W(z)-V(z)]}{z} .
$$

Letting $n \rightarrow \infty$, the steady-state relation (13) is finally obtained.

\section{REFERENCES}

[1] D. O. Reudink, "Spot beams promise satellite communication breakthrough," IEEE Spectrum, pp. 36-42, Sept. 1979.

[2] J. Martin, Communications Satellite Systems. Englewood Cliffs, NJ: Prentice-Hall, 1978.

[3] V. K. Bhargava, D. Haccoun, R. Matyas, and P. Nuspl, Digital Communications by Satellite. New York: Willey, 1981.

[4] Y. Ito, V. Urano, T. Muratani, and M. Yamaguchi, "Analysis of a switch matrix for an SS/TDMA system," Proc. IEEE, vol. 65, pp. 411-419, Mar. 1977.

[5] A. S. Acampora and B. R. Davis, "Efficient utilization of satellite transponders via time-division multibeam scanning," Bell Syst. Tech. J., vol. 57, pp. 2901-2914, Oct. 1978.

[6] T. Inukai, "An efficient SS/TDMA time slot assignment algorithm," IEEE Trans. Commun., vol. COM-27, pp. 1449-1455, Oct. 1979.

[7] G. Bongiovanni, D. Coppersmith, and C. K. Wong, "An optimum time slot assignment algorithm for an SS/TDMA system with variable number of transponders," IEEE Trans. Commun., vol. COM-29, pp. 721-726, May 1981.

[8] G. Bongiovanni, D. T. Tang, and C. K. Wong, "A general multibeam satellite switching algorithm," IEEE Trans. Commun., vol. COM-29, pp. 1025-1036, July 1981.

[9] 1. Gopal, D. Coppersmith, and C. K. Wong, "Minimizing packet waiting time in a multibeam satellite system," IEEE Trans. Commun., vol. COM-30, pp. 305-315, Feb. 1982.

[10] T. Suda, H. Miyahara, and T. Hasegawa, "Optimal bandwidth assignment on up- and downlinks of satellite with buffer capacity," IEEE Trans. Commun., vol. COM-28, pp. 1809-1818, Oct. 1980.

[11] N. Abramson, "The throughput of packet broadcasting channels," IEEE Trans. Commun., vol. COM-25, pp. 117-127, Jan. 1977

[12] J. F. Chang, "A multibeam packet satellite using random access techniques," IEEE Trans. Commun., vol. COM-31, pp. 1143-1154, Oct. 1983.

[13] L. Kleinrock, Queueing Systems, vol. 1. New York: Wiley, 1975.

[14] J. Hsu, "Buffer behavior with Poisson arrival and geometric output processes," IEEE Trans. Commun., vol. COM-22, pp. 1940-1941, Dec. 1974.

[15] W. Chu, "Buffer behavior for batch Poisson arrivals and single constant output," IEEE Trans. Commun., vol. COM-18, pp. $613-678$, Oct. 1970

[16] T. S. Heines, "Buffer behavior in computer communication systems," IEEE Trans. Comput., vol. C-28, pp. 573-576, Aug. 1979.

[17] N. D. Georganas, "Buffer behavior with Poisson arrivals and bulk geometric service," IEEE Trans.. Commun., vol. COM-24, pp. 938-940, Aug. 1976.

[18] H. Kobayashi and A. G. Konheim, "Queueing models for computer communications system analysis," IEEE Trans. Commun., vol. COM-25, pp. 2-28, Jan. 1977.

[19] F. A. Tobagi, "Multiaccess protocols in packet communication systems," IEEE Trans. Commun., vol. COM-28, pp. 468-488, Apr. 1980. 